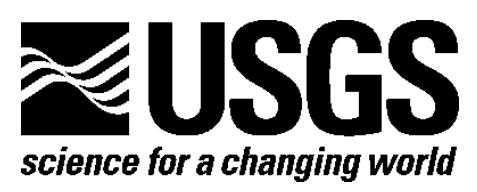

\title{
National GAP Conference 2007-Discussion Groups Report
}

By Joan M. Ratz and Berton Lee Lamb

Open-File Report 2010-1026 


\section{U.S. Department of the Interior \\ KEN SALAZAR, Secretary}

\section{U.S. Geological Survey \\ Marcia K. McNutt, Director}

U.S. Geological Survey, Reston, Virginia 2010

For product and ordering information:

World Wide Web: http://www.usgs.gov/pubprod

Telephone: 1-888-ASK-USGS

For more information on the USGS—-the Federal source for science about the Earth,

its natural and living resources, natural hazards, and the environment:

World Wide Web: http://www.usgs.gov

Telephone: 1-888-ASK-USGS

Suggested citation:

Ratz, J.M., and Lamb, B.L., 2010, National GAP Conference 2007-Discussion groups report: U.S. Geological Survey Open-File Report 2010-1026, 10 p.

Any use of trade, product, or firm names is for descriptive purposes only and does not imply endorsement by the U.S. Government.

Although this report is in the public domain, permission must be secured from the individual copyright owners to reproduce any copyrighted material contained within this report. 


\section{Contents}

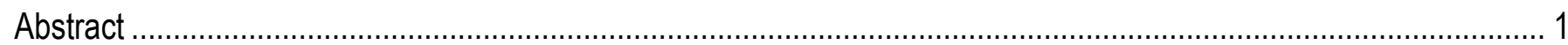

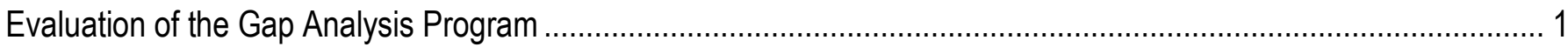

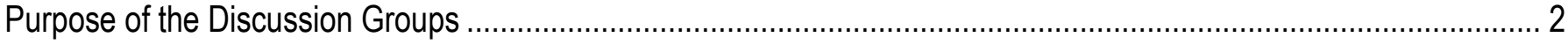

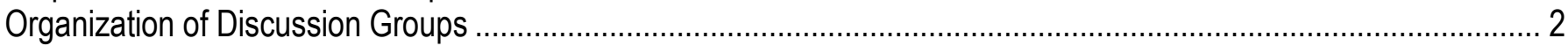

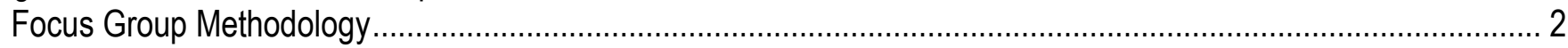

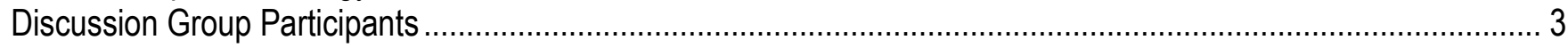

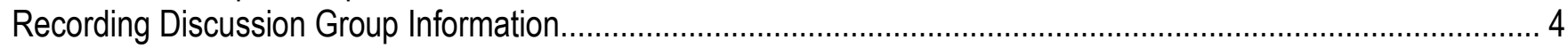

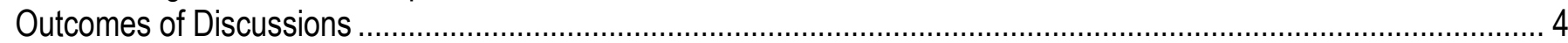

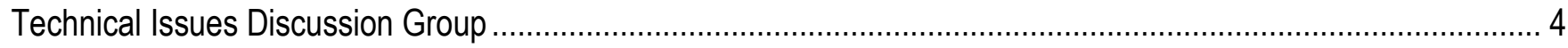

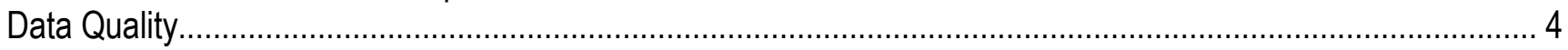

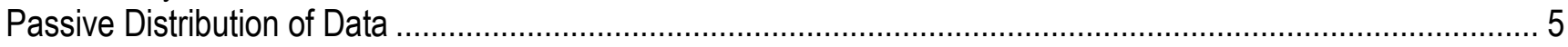

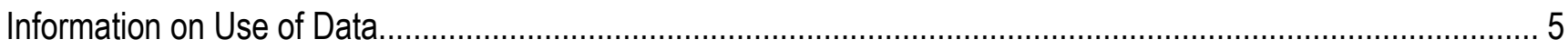

Use of GAP Data for Decisionmaking Discussion Group .......................................................................... 5

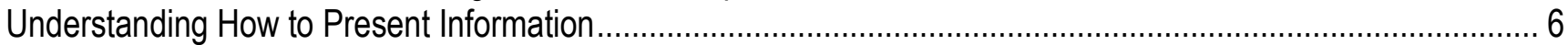

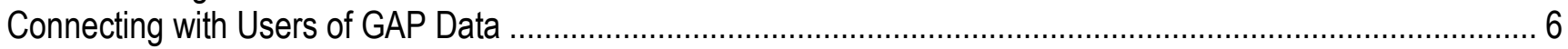

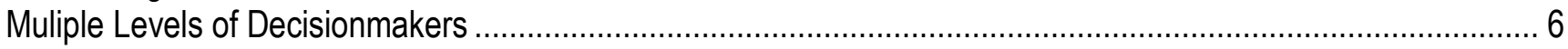

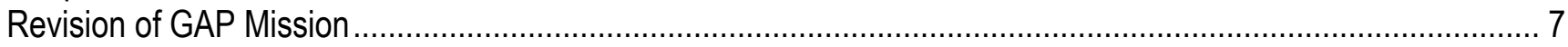

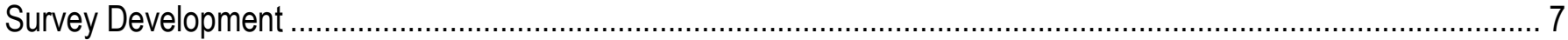

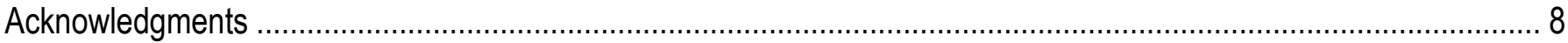

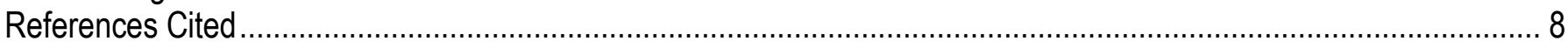

Appendix - Conference Discussion Group Protocol ..................................................................................... 9 


\title{
National GAP Conference 2007-Discussion Groups Report
}

\author{
By Joan M. Ratz and Berton Lee Lamb
}

\begin{abstract}
We led two discussion groups during the 2007 National GAP Conference. These discussion groups provided information to help develop a survey of National Gap Analysis Program (GAP) data users. GAP provides spatial data for use as a framework to make assessments regarding how well biodiversity is protected, and to evaluate what aspects of biodiversity need greater protection. One group discussed technical issues, and the second group discussed the use of GAP data for decisionmaking. Themes emerging from the technical issues group included concerns about data quality, need for information on how to use data, and passive data distribution. The decisionmaking discussion included a wide range of topics including the need to understand presentation of information, the need to connect with and understand users of data, the revision of GAP's mission, and the adaptability of products and data. The decisionmaking group also raised concerns regarding technical issues. One conclusion is that a deep commitment to ongoing information transfer and support is a key component of success for the GAP program.
\end{abstract}

\section{Evaluation of the Gap Analysis Program}

The National Gap Analysis Program (GAP) of the U. S. Geological Survey (USGS) has been operational for more than 15 years. GAP provides spatial data for use as a framework to make assessments regarding how well biodiversity is protected, and to evaluate what aspects of biodiversity need greater protection. Among the program goals are providing "information to the public and those entities charged with land use research, policy, planning, and management," and "build[ing] institutional cooperation in the application of this information to state and regional management activities." To this end, GAP produces and provides the following types of data sets: land cover; predicted distributions of vertebrate species; land stewardship; and analysis data synthesizing the three aforementioned data sets. The original data sets were created at the state level. The state level data sets are available on the GAP website for 48 states and Puerto Rico. Data for Alaska are anticipated in 2010; state data are not available on the GAP site for Alabama. More recent data sets have been assembled at regional data levels. Specifically, regional data are available for three regions: Southwest (Arizona, Colorado, New Mexico, Nevada, Utah); Southeast (Alabama, Florida, Georgia, Kentucky, Mississippi, North Carolina, South Carolina, Tennessee, Virginia); and Northwest (Idaho, Montana, Oregon, Washington, Wyoming). However, not all types of data currently are available for all regions.

In Federal fiscal year 2007, the Policy Analysis and Science Assistance Branch (PASA) of the USGS contracted with the National Gap Analysis Program to conduct a systematic evaluation of GAP. The intent of the evaluation is to assess the impacts that GAP products - the data sets it produces and distributes - have on the individuals who use the products (GAP users) and on conservation of biodiversity. The main component of such an evaluation is a data collection process using a survey 
designed to measure the extent to which GAP is achieving its stated goals (Rossi and others, 2004). The results of this survey will be used by GAP administrators and staff to evaluate past performance of GAP and to strategically plan changes to the current approach. Changes in the existing approach to developing and providing data may be necessary to best meet the needs of current GAP users and expand the number of GAP users. The survey is the key piece of the evaluation project and it must be carefully designed. To support and inform survey development, we collected information from several outlets. We surveyed the published literature for studies making use of GAP data, reviewed the literature on use of geographic information system (GIS) data for decisionmaking and related topics, and conducted two discussion groups that included GAP users. In this report, we focus on the themes arising from the two discussion groups held during the 2007 National GAP Conference. These discussion groups were held for the purpose of including topics identified by GAP users on the survey.

\section{Purpose of the Discussion Groups}

We needed more information about two topics of particular interest before we could develop effective questions to include on the survey: (1) technical issues encountered when using GAP data, and (2) use of GAP information in decisionmaking processes. While GAP staff had their perceptions of what might be of concern to GAP users in these areas, we could more accurately determine the users' viewpoints by asking a group of individuals who are informed about GAP but not part of the GAP staff. We led two small groups in targeted discussions on September 10 and September 12, 2007 during the National GAP Conference in Asheville, North Carolina.

\section{Organization of Discussion Groups}

\section{Focus Group Methodology}

In organizing these discussion groups we followed the general principles of focus group methodology (see Greenbaum, 1993; Morgan 1993). For practical reasons, we convened our groups for 60 minutes, although focus groups commonly last between 90 and 120 minutes (Greenbaum, 1993). We (Ratz and Lamb) were both present and participating in the groups with Ratz taking the lead in group moderation. The moderator followed a written moderator guide to set the context and the key issues for discussion (Greenbaum, 1993; Knodel, 1993).

Each group was directed to discuss a particular topic - one group was directed to discuss the technical issues in using GAP data, and the second group was directed to discuss use of GAP data in decisionmaking. Topics for discussion should be limited and defined in focus groups (Knodel, 1993). Focus group methodology is designed to obtain more detailed information about a specific topic, rather than less detailed information about a broad range of topics. However, the interactive nature of group discussions can lead to digressions and diversions from the topic of interest. The reason for limiting the topics for discussion is to give direction to the group so that the intent of the focus group is fulfilled. At the beginning of each group session, the topic for the group was defined and a few questions were posed to the group to get them started. Participants were told that they were free to pose their own questions for discussion as long as the questions related to the overall topic of discussion. The technical issues group was asked to discuss the issues involved in using GAP data. Technical issues could relate to characteristics of the data, or to software or hardware issues. The group was asked to what degree do technical issues create barriers to using GAP data and how the barriers affect the use of GAP data for the preservation of biodiversity. The decisionmaking group was asked to discuss the issues involved in transferring knowledge from GAP products to decisionmakers and how they get the information into the 
hands of people who make decisions. They also were asked to describe how GAP-based information currently is used in decisionmaking processes and how it can best be communicated to those in decisionmaking positions. The conference discussion group protocol includes the moderator guide and the questions posed to group participants; this protocol is provided in the Appendix to this report.

Social desirability can affect the comments that people make when they participate in focus groups (Albrecht and others, 1993). Socially desirable responding_providing responses that are perceived as being more socially acceptable - is a threat to the validity of focus group data. Situations that might induce this type of participation should be avoided. We believed that a possibility of social desirability existed because the population of GAP users is socially networked. For example, individuals who work for state natural resource agencies likely are to have contact with local government agencies and conservation nonprofit organizations operating within the same state. Because the participants in the discussion group are not in the position of being supervised by GAP staff, their comments were unlikely to result in any personal benefit. Any socially desirable effect likely to be present would have been driven by the desire to maintain an image around colleagues, not motivated by the desire to influence personal outcomes. For this reason, we did not believe that social desirability was a strong threat. To minimize social desirability and to protect the identity of group participants, we asked GAP staff to stay away from the focus groups while they were in session. Participants were told that only themes would be reported from the focus group and that their names would not be attached to any comments. Based on the tenor of the discussions, we believe that socially desirable responding was not an issue in either focus group.

\section{Discussion Group Participants}

A common recommendation in traditional focus group methodology is to have group members be similar with respect to demographics because this is believed to produce more in-depth information (Greenbaum, 1993; Morgan, 1993). A more contemporary position is that focus groups should be designed to best achieve the purpose of the research project (Morgan, 1996). For our purposes, it would work to our advantage to have a diverse group of participants. To develop survey questions, we need to understand the perspective of multiple types of GAP data users.

We ran a random selection procedure within a sampling frame of a convenience population. We imported the conference registration list into SPSSC software and used a random case selection function to identify a sample. Approximately two weeks before the conference, we sent the selected sample an email inviting them to participate in a discussion group. In the email we explained the reason for the discussion groups, described the topics for discussion, listed the dates and times for the sessions, and invited the email recipient to respond if he or she would like to participate in a discussion group. In the email, the discussion groups were described as "invitation only" and were listed in the conference program with that designation. Participants self-selected the discussion topic. The decisionmaking discussion group filled quickly. We sent an additional mailing at a later date to an additional set of randomly selected individuals to solicit enough participants for the technical issues group. The technical issues discussion group was held on Monday night, the first night of the conference, and may have had a lower response because some people did not arrive until Monday night or later.

We sent invitations to 43 individuals who were registered for the conference (34 percent of conference registrants). Twenty-five individuals agreed to participate in a discussion group; this is a participate rate of 58 percent. This sample for the discussion groups cannot be considered a random or representative sample of the entire population of GAP data users. However, our goal was to obtain information to use in development of a survey. We needed individuals who were knowledgeable about GAP data and willing to provide input about the identified topics. Our sample satisfied that purpose. 
The technical issues discussion group had 10 participants and one no-show. The group included academic faculty and employees of federal and state agencies. There were six males and four females in this group. Based on the registration for the conference, this attendance represented 8 percent of conference registrants. The decisionmaking group had 13 participants, one no-show, and one gatecrasher. The group included individuals employed by universities, federal and state agencies, and nonprofit organizations. There were nine females and five males in this group. The 14 individuals in this group represented 11 percent of conference registrants.

\section{Recording Discussion Group Information}

We used note takers to record information from the group discussions. We opted not to record the group discussions for privacy-related as well as practical reasons. Regulations that apply to data collection by Federal agencies limit the type and amount of personal information that can be collected. There also are regulations that apply to the secure storage of personal information. Additionally, the GAP user group is characterized by social relationships. There are professional connections among GAP users. Therefore, we believed there was a heightened need to protect privacy of the participants and to exclude identity from the data collection. Beyond privacy issues, recording and transcribing the focus groups would have increased the cost of this process. Recording the groups for transcription would have required that we rent equipment. Small hand-held recorders such as we have used in the past for recording individual interviews lack sufficient range to record all group members. Transcription is costly. Given that we were interested in general thematic content to guide development of survey questions and that we were not doing an in-depth analysis of the focus group discussion, we determined that the cost of recording and transcribing outweighed the contribution of this approach.

\section{Outcomes of Discussions}

Both groups included a diverse mix of participants in terms of organizational affiliation and gender. Because we randomly selected individuals from a list of conference registrants to invite and allowed them to self-select in which group they would participate, we did not have control over the demographic characteristics of the groups. We sought to hear from a variety of types of GAP users because GAP data potentially can be used by so many different groups. We heard a variety of concerns and comments; we have distilled the comments into thematic categories.

Overquantifying the results of focus groups is considered an analytical error (Greenbaum, 1993). Therefore, we do not attempt to quantify the "main" or "primary" themes arising from each group. We reviewed the information we recorded from each group and determined the emergent themes.

Comments that were similar in content were considered to be from a common theme. A single comment was not considered a theme. We report the themes emerging from the two discussion groups. We report themes using the words of participants as much as possible.

\section{Technical Issues Discussion Group}

The themes emerging from the technical issues discussion included: data quality concerns (outdated, inconsistent quality, and issues of scale and resolution), passive distribution of data, and need for information on how to use data.

\section{Data Quality}

Many specific issues are included in the theme of data quality. GAP data are viewed as being too old, especially since in some areas the only data available are from the state projects. The stewardship 
data set was singled out as being the most difficult to keep current. There is turnover in private and leased land that could affect stewardship mapping. Lands are being fragmented rapidly which leads to change in ownership and conservation status. There has been inconsistency in data quality, although this perception may be a hold over from the state projects. Nonetheless, the participants believe this perception has created a reputation problem for GAP. Because reputation can affect future use of data, regardless of whether there is truth behind the reputation, we include questions about GAP reputation on the survey. We include a series of specific questions addressing multiple aspects of data quality on the survey that will help us better describe issues with the data.

The issues of scale and resolution are multiple. Several participants noted that GAP data is not a scale that can be used for local projects. Yet, funding often is more available for local projects. Many people work at a smaller level rather than the landscape level, but GAP data are too coarse for work in a smaller frame. There is a conceptual disconnect forming for GAP. The push is to go to a larger national scale, but will the data be at an appropriate scale for the people who really need it who are working on smaller projects? Participants noted a need for GAP to define their target audience. Given the other comments made regarding appropriateness of scale, we conclude that once the target audience is defined GAP may need to consider scaling the data to the needs of that group. The issue of whether or not the scale of GAP data meets the needs of the individual users will be included on the survey. The survey data will allow us to conclude the extent to which GAP data is meeting the needs of the users.

\section{Passive Distribution of Data}

GAP data sets are distributed in a passive manner with no connection between GAP staff and the recipient of the data. No instructions accompany the data. GAP users will have varied levels of GIS competence and GAP needs to provide training and other support for users. Previously GAP data were generated in partnership with states so the data were provided in process in which users were more actively engaged. With the change to regional data sets, the states are less involved and have less buy-in to use the data. Based on concerns expressed by group participants, GAP should keep in mind that not all users will have up-to-date computers and software when considering how to distribute data. The survey includes questions on how users acquire data, how they would like to acquire data, and other information they need from GAP about data use.

\section{Information on Use of Data}

The need for information is tied closely with the issue of passive distribution of data sets. Not only is there a need for information and education on how to use GAP data, but there is a need for educating users on limitations of GAP data and how a product generated based on GAP data will be affected. Users need to understand how accuracy is assessed in GAP data sets. Many users, based on the assumption of varying degrees of GIS competence, may not understand metadata or how GAP data is limited based on its underlying models. Participants suggested that understanding and communicating about limits is important because maps are viewed by others as infallible. Decisionmakers will rely on the models generated by GAP data but at the same time researchers question the underlying data and modeling strategies.

\section{Use of GAP Data for Decisionmaking Discussion Group}

In the decisionmaking discussion group, there were some group dynamics that were not well controlled by the moderator. The group discussion was dominated by a few strong participants including the gatecrasher. The analytical issue that arises from a group that is dominated by a few participants is 
that too much emphasis is placed on the input of a few and the range of opinions represented by the group may not be not represented as well (Greenbaum, 1993). The outcomes of the decisionmaking discussion group should be considered keeping this caveat in mind. However, our goal in conducting the focus group was to collect information from which to develop survey questions. We do not believe that the strong personalities prevented us from obtaining the information we were asking participants to provide. The survey data will yield information as to whether the issues raised by the more dominant participants are representative of the opinions of GAP data users as a group.

The themes emerging from the decisionmaking discussion group included: (1) need to understand presentation of information, (2) need to connect with end users of data and understand their needs, (3) consider that decisionmakers at several levels influence whether GAP data are used, (4) questions raised about GAP revising its mission to include other types of data (socioeconomic), and (5) make data more adaptable and provide other more adaptable products.

\section{Understanding How to Present Information}

Participants noted that the marketing aspect of GAP data has been neglected. In order to effectively market the data, participants suggest that program staff need to understand what information to present and how to present it. It is important to have the right person do outreach. Stories and case studies are effective methods to demonstrate how GAP data can be used. The information should be kept simple. Participants stated that a key component of marketing is to understand the target market for GAP. They did not elaborate on the target market but commented that there likely are varied users and that GAP does not seem to be quite meeting the needs of any of them. The survey will include a series of questions about the data needs of GAP users and what kind of supporting information they prefer.

\section{Connecting with Users of GAP Data}

In order to effectively market GAP data, GAP needs to understand more of the characteristics of the users of GAP data. Developing connections with users of GAP data will take time. There used to be a natural connection between GAP and its users because users were enlisted as cooperators to develop the data sets. Since the shift to regional level data sets, GAP has lost some connection to the user group. Participants commented that developing relationships with users will benefit GAP by helping to define what questions need to be answered by users. The questions drive the data requirements. Connecting with end users of data will help GAP understand what their users really need in terms of data.

Part of the discussion focused on the relationship between GAP understanding what users really need and marketing GAP. The information provided to "sell" the data must be directly relevant to the user's needs. Participants noted that some users may not have the GIS expertise to easily use GAP data and will need more support from GAP program staff. In the past, state GAP projects ended (ran out of funding) before the agencies were trained to use the data. While connecting GAP to its users is a goal beyond the means of a survey, the survey will result in information that GAP can use in future efforts to connect with users.

\section{Muliple Levels of Decisionmakers}

One of the stated goals of this evaluation of GAP is to determine the effect of the program on conservation of biodiversity. The tendency in discussing decisionmakers has been to focus on those individuals who make on-the-ground decisions about conservation. However, the group participants believe GAP should consider that there are people at multiple levels along that decision chain who will influence whether and how GAP data are used. Participants noted that the GIS professional who selects 
the data sets is a decisionmaker who has a large effect on use of GAP data. There will be several questions on the survey about the types of decisions made by the survey respondent and about how information is disseminated to other decisionmakers.

\section{Revision of GAP Mission}

The issue of revising the GAP mission was discussed. Some participants suggested additional information, such as socioeconomic and quality of life indicators, and demographics, could be incorporated into GAP data to set it apart from some other data sets. GAP needs to define itself as a unique program and establish its niche. Discussion group participants noted that the information presented by GAP staff at the conference gave different interpretations and emphases on the mission of GAP. Survey questions include assessments about how well GAP is meeting its current mission and reactions to potential changes to that mission.

Other technical issues comments crept into the decisionmaking discussion. The technical issues mentioned were data quality issues that have impacted the reputation of GAP data, inconsistency between states and lack of continuity in data sets.

Although we stated at the beginning of each discussion group that aquatic GAP data were not going to be part of the discussion, comments about aquatic GAP were made by participants: aquatic GAP data sets have poor metadata; aquatic GAP data are used frequently in some agencies; aquatic GAP should be integrated with terrestrial GAP.

\section{Survey Development}

The evaluation of GAP is intended to be a formative evaluation. That is, the information is collected and analyzed in the context of learning how to improve the program to better achieve its stated goals and better serve the public. Program evaluation involves collecting, analyzing, and interpreting data in one or more of the following five domains (Rossi and others, 2004): (1) the need for the program, (2) the program's design, (3) its implementation and service delivery, (4) its impact or outcomes, and (5) its efficiency. The survey that is the core of the GAP assessment includes the domains of need for the program (user needs), implementation and delivery, and impact. The survey questions were written based on comments from GAP staff, the themes from the GAP Conference discussion groups, comments made in published articles that used or referenced GAP data, and the literature on use of GIS data.

The user needs assessment portion focuses on technological and educational needs of GAP data users. The technical issues discussion group and the decisionmaking group underscore the importance for GAP to understand the needs of its users. GAP needs to understand what the users are using the data for in order to determine the appropriate scale of data. They also need to understand what questions the users are trying to answer with the data. Questions addressing characteristics of users and their data needs are included in the user needs assessment. The technical issues group noted that there is no instruction that accompanies GAP data. Questions on the survey will address the education needs of users. To address the concern raised that not all GAP users have up-to-date software and hardware, we incorporated some questions about the technological requirements of users.

Assessments of implementation and service delivery focus on how the program is carried out; this is also known as process evaluation (Rossi and others, 2004). The primary topic in this domain is user satisfaction with the process used by the GAP program to produce and deliver data, and outreach to facilitate data use. User satisfaction with the data is included and addresses the concerns expressed in the discussion groups regarding data quality. Given the observation made about decisionmakers being at 
multiple levels in a decision chain, we also included questions about the decision-making power of the user.

An impact assessment is conducted to evaluate the outcomes of a program and the effect that the outcomes have; specifically, the intent is to determine if the program is having the intended effect. The impacts assessed in this evaluation are proximal (immediate effect on the user) and distal (users' estimation of overall effects). User satisfaction also can be used in impact assessments. The technical issues group discussed issues with GAP's reputation and questions regarding user judgments of GAP data are part of the impact assessment. User perception of GAP meeting its overall goals and the overall impact of GAP relate to the issue raised regarding a revision of the GAP mission.

This survey will be sent to users of GAP data who are employed by government at the federal, state, and local levels, educational institutions, nonprofit organizations, and by international and tribal governments. Because the survey participants are not limited to federal employees, the survey is subject to an information collection request process under the purview of the Office of Management and Budget (OMB). As part of that process, the survey must be made available for public review and comment. Notices regarding this survey are published in the Federal Register.

We greatly appreciate the contribution of time and energy of the conference discussion group participants. We would not have been able to acquire the information without their assistance. The themes generated from the conference discussion groups and the questions generated based on those themes will enhance the evaluation project and provide the Gap Program with the information it needs.

\section{Acknowledgments}

This project was commissioned by the Gap Analysis Program of the U.S. Geological Survey. We would like to thank Dr. Jacob Hautaluoma and Dr. Lori Peek for their helpful and thoughtful reviews of this report.

\section{References Cited}

Albrecht, T.L., Johnson, G.M., and Walther, J.B., 1993, Understanding communication processes in focus groups, in Morgan, D.L., ed., Successful focus groups: advancing the state of the art: Newbury Park, Calif., Sage Publications, p. 51-64.

Greenbaum, T.L., 1993, The handbook for focus group research: New York, Lexington Books.

Knodel, J., 1993, The design and analysis of focus group studies, in Morgan, D.L., ed., Successful focus groups: advancing the state of the art: Newbury Park, Calif., Sage Publications, p. 35-50.

Morgan, D.L., ed., 1993, Successful focus groups: advancing the state of the art: Newbury Park, Calif., Sage Publications.

Morgan, D.L., 1996, Focus groups: Annual Review of Sociology, v. 22, p. 129-152.

Rossi, P.H., Lipsey, M.W., and Freeman, H.E., 2004, Evaluation: a systematic approach (7th ed.):

Thousand Oaks, Calif., Sage Publications. 


\section{Appendix - Conference Discussion Group Protocol}

1. Introductory comments by Lamb

1. Overall GAP evaluation project

2. Introduce Ratz

2. Comments by Ratz

1. Why do a conference discussion group?

1. Issues currently defined in a broad fashion.

2. Writing survey questions without additional information would be less effective.

3. Results from discussion can be used to inform survey questions; determine what concerns should be investigated more fully in our evaluation project.

2. Ground rules for discussion

1. No names recorded.

2. Please be as honest as possible; otherwise feedback will not be as useful.

3. Topic specific comments to begin the discussions: These are some general topics we are interested in, participants are free to pose questions for discussion as long as they relate to the overall topic of discussion.

- MONDAY: Technical Issues

- What are technical issues involved in using GAP data?

- Technical issues could relate to characteristics of the data, or to software or hardware issues.

- To what degree, if any, do technical issues create barriers to using GAP data?

- How do these barriers affect the use of GAP for the preservation of biodiversity (GAP overall goal)

- WEDNESDAY: Decisionmakers

- What are the mechanics of transferring knowledge from the GAP products to decisionmakers?

- How do we get it into the hands of people who can make a difference?

- How is GAP-based information currently used in decisionmaking processes?

- How can GAP-based information be best communicated to those in decisionmaking positions? 
Publishing support provided by:

Denver Publishing Service Center

For more information concerning this publication, contact: Center Director, USGS Fort Collins Science Center

2150 Centre Ave., Bldg. C

Fort Collins, CO 80526-8118

(970)226-9398

Or visit the Fort Collins Science Center Web site at: http://www.fort.usgs.gov/ 